\title{
Anti-CTLA-4 monoclonal antibody improves efficacy of the glyceraldehyde-3-phosphate dehydrogenase protein vaccine against Schistosoma japonicum in mice
}

\author{
Chun-lian Tang ${ }^{1} \cdot$ Jin-feng Yang ${ }^{1} \cdot$ Qun Pan ${ }^{1} \cdot$ Rong-hui Zhang ${ }^{1} \cdot$ Ya-ping Xie ${ }^{1} \cdot$ Ying Xiong $^{1} \cdot$ Hong-hua Zhou $^{1}$
}

Received: 13 January 2019 / Accepted: 24 May 2019 / Published online: 5 June 2019

(C) Springer-Verlag GmbH Germany, part of Springer Nature 2019

\begin{abstract}
Schistosomiasis is a devastating disease caused by Schistosoma infection. Glyceraldehyde-3-phosphate dehydrogenase (GAPDH) has emerged as a candidate vaccine component against Schistosoma japonicum, but only confers partial protection. Cytotoxic T lymphocyte antigen-4 (CTLA-4) regulates T cell activation and shows negative effects on vaccine-induced immune protection; however, its potential influence on the protective effects of a GAPDH vaccine against S. japonicum and the underlying mechanism remain unclear. In this study, we established a mouse model of $S$. japonicum infection, and the mice were randomly divided into uninfected, infected control, anti-CTLA-4 monoclonal antibody (anti-CTLA-4 mAb), GAPDH, and GAPDH combined with anti-CTLA-4 mAb groups to compare the protective effects against infection and the consequent tissue damage. The worm reduction rate in the GAPDH-treated infected mice was $26.58 \%$, which increased to $54.61 \%$ when combined with anti-CTLA- $4 \mathrm{mAb}$. The frequency of regulatory T cells (Tregs) was significantly higher in the anti-CTLA- $4 \mathrm{mAb}$ group and was lower in the GAPDH group. However, both anti-CTLA-4 mAb and GAPDH elevated the levels of the cytokines IFN- $\gamma$, IL2, IL-4, and IL-5 in the spleens of infected mice, and their combination further enhanced cytokine production. The diameter of egg granuloma in the anti-CTLA-4 mAb group and combined treatment group increased significantly compared to that of the other groups. These results suggest that anti-CTLA-4 mAb can be used as an adjuvant to enhance the immune protection of the GAPDH vaccine via inducing the Th1 immune response, although this comes at the cost of enhanced body injury.
\end{abstract}

Keywords Schistosoma japonicum · Glyceraldehyde-3-phosphate dehydrogenase $\cdot$ Regulatory T cells $\cdot$ Cytotoxic T lymphocyte antigen-4 · Granuloma

\section{Introduction}

Schistosomiasis is a fatal disease representing a significant public health threat and economic burden worldwide. Eggs of Schistosoma are the most important pathogenic factors, leading

Chun-lian Tang and Jin-feng Yang contributed equally to this work.

Section Editor: Ramaswamy Kalyanasundaram

Ying Xiong

xiongying_123@126.com

Hong-hua Zhou

zhouhonghuatang@163.com

1 Wuchang Hospital, Wuhan University of Science and Technology, No. 116 Yangyuan Street, Wuhan 430063, China to late symptoms that are difficult to resolve, such as liver fibrosis caused by egg deposition in the liver of the host (Sun et al. 2017). Praziquantel is the first choice for the treatment of schistosomiasis, but has no preventive effect. Therefore, development of an effective vaccine is essential for schistosomiasis control. As the key enzyme in glycolysis, glyceraldehyde-3-phosphate dehydrogenase (GAPDH) has been proposed as a suitable candidate component of a Schistosoma japonicum subunit vaccine (Argiro et al. 2000). Perez-Casal and Potter (2016) reported that schistosome GAPDH confers partial resistance to schistosomiasis, emerging as a candidate subunit vaccine for inducing protective immunity. The recombinant GAPDH from $S$. japonicum (rSjGAPDH) was first cloned and molecularly characterized as a candidate vaccine (Waine et al. 1993). Several decades later, the peptidases of Schistosoma mansoni CL3 and CB1 were combined with GAPDH, which increased the protective levels against $S$. mansoni infection challenge up to $70-76 \%$, while 
the number of eggs and their viability decreased significantly (Tallima et al. 2017). Thus, GAPDH appears to be an effective agent for infection prevention; however, the mechanism of this protective effect remains unclear. This mechanism has been proposed to be associated with regulatory T cells (Tregs). Tang et al. (2017) confirmed that the inadequate immune protection of the glutathione-S-transferase (GST) vaccine may be related to the induction of Tregs by the vaccine, and an anti-CD25 monoclonal antibody $(\mathrm{mAb})$ improved the protective efficacy of the GST vaccine by blocking Tregs. Cimetidine was also suggested to improve the protective effect of this vaccine by reducing the percentage of Tregs ( $\mathrm{Li}$ et al. 2011).

Tregs comprise various $\mathrm{T}$ cell subsets with inhibitory properties, expressing phenotypic markers such as the alpha chains of the interleukin (IL)-2 receptor, cytotoxic T lymphocyteassociated protein 4 (CTLA-4), glucocorticoid-induced tumor necrosis factor receptor (GITR), forkhead box protein 3 (Foxp3), and lymphocyte activation gene 3 (LAG3) (Pietruczuk et al. 2012). CTLA-4, a member of the immunoglobulin-related receptor family, is responsible for various aspects of $\mathrm{T}$ cell immune regulation (Rowshanravan et al. 2018), including its most important function in inhibiting $\mathrm{T}$ cell activation. Mice with a $\mathrm{Ctla} 4$ gene deficiency show lymphocyte proliferation and infiltration to multiple organs, and die 3-4 weeks after birth (Waterhouse et al. 1995). CTLA4 has also been demonstrated to play an important role in vaccine design and can exert protective effects on its own. Indeed, vaccine-induced protective immunity can be regulated by the induction of co-inhibitory ligands such as CTLA-4, which induce the depletion and non-proliferation in antigenexperiencing T cells (Gannavaram et al. 2016). For example, the development of an immune response to a tetanus toxoid vaccine was found to be altered by a submicroscopic malaria infection in Colombian pregnant women, and CTLA-4 expression levels were lower in the infected group (ÁlvarezLarrotta et al. 2019). Thus, antibodies that bind to CTLA-4 have great potential in the treatment of chronic infections, especially in combination with therapeutic vaccines (Dyck and Mills 2017); however, the role of CTLA-4 in a vaccine of $S$. japonicum GAPDH has not yet been investigated. Accordingly, in this study, we explored the effect of CTLA4 on the protective efficacy of a GAPDH vaccine and its underlying mechanism using an anti-CTLA- 4 mAb. Mice were infected with cercariae of $S$. japonicum and then treated with $\mathrm{rSjGAPDH}$, anti-CTLA-4 mAb, their combination, or phosphate-buffered saline (PBS) as a control. The effectiveness of the treatments was evaluated based on reduction in worm and egg burdens, and histopathology of the liver. The immune response was assessed by evaluating the Tregs population in the splenic lymphocytes and the production of cytokines in the spleens of the mice of the different groups. These results can lay the foundation for an effective strategy of vaccine development for schistosomiasis.

\section{Materials and methods}

\section{Animals and parasites}

Forty female BALB/c mice aged 6-8 weeks were purchased from Hubei Province Center for Disease Control and Prevention, China. The mice were equally and randomly divided into the uninfected, infected control, anti-CTLA-4 mAb-treated, GAPDH-treated, and combined groups. Oncomelania hupensis infected with S. japonicum was provided by Jiangsu Provincial Institute of Parasitic Disease Control, China. The cercariae escape from positive $O$. hupensis under light.

\section{Experimental schedule}

The GAPDH used in this study is a recombinant protein prepared by our laboratory. Briefly, Sj-GAPDH full-length cDNA was amplified from the adult $S$. japonicum cDNA library using specific primers (forward: 5'-CCGGAATTCATGTC GAAGGCTAAGGTTG-3'; reverse: 5'-CCGC TCGAGTCAATTTCCGTTTAATGCAG-3'). The polymerase chain reaction products of approximately 705 base pairs were ligated into the expression vector PET28a $(+)$ (Novagen, Madison, WI, USA). The recombinant $\mathrm{Sj}$-GAPDH protein (approximately $28 \mathrm{kDa}$ ) was expressed by $1 \mathrm{mM}$ isopropyl- $\beta$-thiogalactopyranoside, purified using Ni-NTA Agarose (QIAGEN, Hilden, Germany), and quantified with a BCA protein Assay Kit (Beyotime, Shanghai, China) according to the manufacturer's protocol.

The mice in the GAPDH and combined groups were immunized with $\mathrm{rSjGAPDH}(100 \mu \mathrm{g})$, whereas $100 \mu \mathrm{l}$ PBS was used in the remaining three groups. Immunization was boosted with the same volume at 2 -week intervals. Two weeks after the last immunization, the mice in the four infected groups were percutaneously infected with $40 \pm 1$ cercariae of $S$. japonicum per mouse. The mice in the anti-CTLA- $4 \mathrm{mAb}$ and combined groups were injected intraperitoneally with anti-CTLA-4 mAb $(300 \mu \mathrm{g}) 2$ weeks post-infection; the same volume of PBS was used as a control. All mice ( 8 mice per group) were sacrificed 5 weeks after infection ( 3 weeks after anti-CTLA-4 mAb administration) for assessment. The treatment regimen is schematically depicted in Fig. 1.

\section{Worm-burden and egg-burden assessment}

To detect the protective efficacy of rSjGAPDH and antiCTLA-4 mAb alone or in combination, the mice in the four infected groups were killed, and adult worms were obtained by portal vein infusion according to the method described by Ruppel et al. (1990). The number of worms was counted under an anatomical microscope, and the reduction rate of the worms was calculated. To obtain the egg load, the livers in all 
Fig. 1 Schematic of the experimental schedule
Mouse

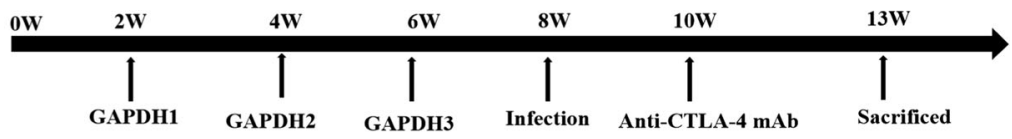

infected mice were removed and digested by $5 \%$ potassium hydroxide. Counts were conducted in $1 \mathrm{ml}$ samples, and the liver egg reduction rate was calculated according to the method described by Cheng et al. (2008). The remaining livers were used for histopathological evaluation of the granuloma. The experiment was carried out in triplicate.

\section{Flow cytometry}

To evaluate the frequency of Tregs, single splenic lymphocytes of mice were prepared according to the method of Mo et al. (2007). In brief, the spleen was gently ground and filtered through a 200-mesh filter cloth. Red cells in the cell pellet were cleaved by erythrocyte lysate (Beyotime, China) and the cells were resuspended in RPMI-1640 medium and grouped at a density of $5 \times 10^{6}$ cells for flow cytometry analysis. The cells were stained with fluorescein isothiocyanateconjugated anti-mouse CD4, allophycocyanin-conjugated anti-mouse CD25, and phycoerythrin-conjugated anti-mouse Foxp3 (all from the Regulatory $\mathrm{T}$ cell staining Kit for mice, eBioscience). All stained cells were analyzed on a Calibur flow cytometer (Becton Dickinson, Mountain View, CA).

\section{Cytokines in the culture supernatant of murine spleen cells}

Single splenocyte suspensions were prepared as described above, and then $5 \times 10^{6}$ cells from the spleen suspension of each mouse were transferred to 24-well culture plates (Corning, USA) and incubated with $5 \mu \mathrm{g} / \mathrm{ml} \mathrm{S}$. japonicum soluble egg antigen or medium alone (all from Sigma). The supernatant was collected after $72 \mathrm{~h}$ of culture $\left(37^{\circ} \mathrm{C}\right.$ in $5 \%$ $\mathrm{CO}_{2}$ ) for enzyme-linked immunosorbent assay to measure the levels of the cytokines IL-2, interferon (IFN)- $\gamma$, IL-4, and IL-5 in the supernatant according to the manufacturer instructions of the respective kits (eBioscience).

\section{Histopathological evaluation}

The partial liver lobe of the mice in the four infected groups was fixed in $10 \%$ formalin and processed into paraffin blocks. Histopathological observation was conducted on 5- $\mu \mathrm{m}$-thick sections by hematoxylin and eosin (H\&E) staining. Typical $\mathrm{H} \& \mathrm{E}$-stained liver sections of each mouse were scanned at $\times$ 200 magnification under a compound microscope.

\section{Statistical analysis}

All data are expressed as the mean \pm standard deviation and differences among groups were determined with analysis of variance using SPSS 19.0 software. A $P$ value of $<0.05$ indicated a significant difference.

\section{Results}

\section{Effect of anti-CTLA-4 mAb on the protective efficacy of $r S j G A P D H$ in mice}

Five weeks after infection, the numbers of worms and eggs in the antibody group and in the GAPDH group were significantly lower than those of the infected control group $(P<0.05)$, but were reduced to a much greater extent in mice that received the combined GAPDH and anti-CTLA- 4 mAb treatment than those in mice treated with either agent alone (Table 1).

\section{Percentages of Tregs in splenocytes of the infected mice}

The transcription factor Foxp3 is typically expressed in Tregs, and Foxp3-specific $\mathrm{T}$ cells can specifically recognize Tregs (Larsen et al. 2013). Therefore, we defined triple-positive $T$ cells $\left(\mathrm{CD} 4^{+} \mathrm{CD} 25^{+} \mathrm{Foxp}^{+} \mathrm{T}\right.$ cells) as Tregs in the present study
Table 1 Immunoprotection of the four infected groups

\begin{tabular}{lllll}
\hline Group & Worm burden & $\begin{array}{l}\text { Worm reduction } \\
\text { rate }(\%)\end{array}$ & $\begin{array}{l}\text { Number of eggs } \\
\text { per gram liver tissue }\left(\times 10^{3}\right)\end{array}$ & $\begin{array}{l}\text { Liver egg } \\
\text { reduction rate }(\%)\end{array}$ \\
\hline Infected control & $28.29 \pm 3.42$ & & $26.31 \pm 8.34$ & \\
Anti-CTLA-4 mAb & $23.02 \pm 2.64$ & $18.64^{* \#}$ & $20.90 \pm 6.27$ & $20.56^{* \#}$ \\
GAPDH & $19.73 \pm 2.34$ & $30.25^{*} \#$ & $17.46 \pm 5.68$ & $33.63^{* \#}$ \\
Combined & $11.53 \pm 1.82$ & $59.36^{*}$ & $9.88 \pm 4.78$ & $62.45^{*}$ \\
\hline
\end{tabular}

Data are expressed as mean $\pm \mathrm{SD}$ from three independent experiments (8 mice per group) $* P<0.05$ compared to the infected control group; $\# P<0.05$ compared to the combination group 
based on flow cytometry analysis. Compared with those of the uninfected group, the infected mice showed significantly higher percentages of Tregs in the splenocytes (Fig. 2). In the infected background, compared with those of the infected group, the percentages of Tregs in the GAPDH-treated group were significantly lower, whereas they were significantly higher in the anti-CTLA-4 mAb-treated group $(P<0.05)$. The frequency of Tregs in the combination group was also significantly higher than that in the GAPDH group $(P<0.05)$. However, there was no significant difference in Treg percentages between the combined group and uninfected group.

\section{Cytokine production by splenocytes}

S. japonicum schistosomula is the main target of vaccine immunization, and it typically takes 4 weeks for the development of schistosomula to mature adult worms. Therefore, we observed the changes of cytokines at 5 weeks after infection. Figure 3 shows that the levels of the Th1-related cytokines IFN- $\gamma$ (Fig. 3a) and IL-2 (Fig. 3b), as well as the levels of the Th2-related cytokines IL-4 (Fig. 3c) and IL-5 (Fig. 3d) were significantly higher in the mice of the infected groups. Consistently, both the antibody and GAPDH-treated infected a
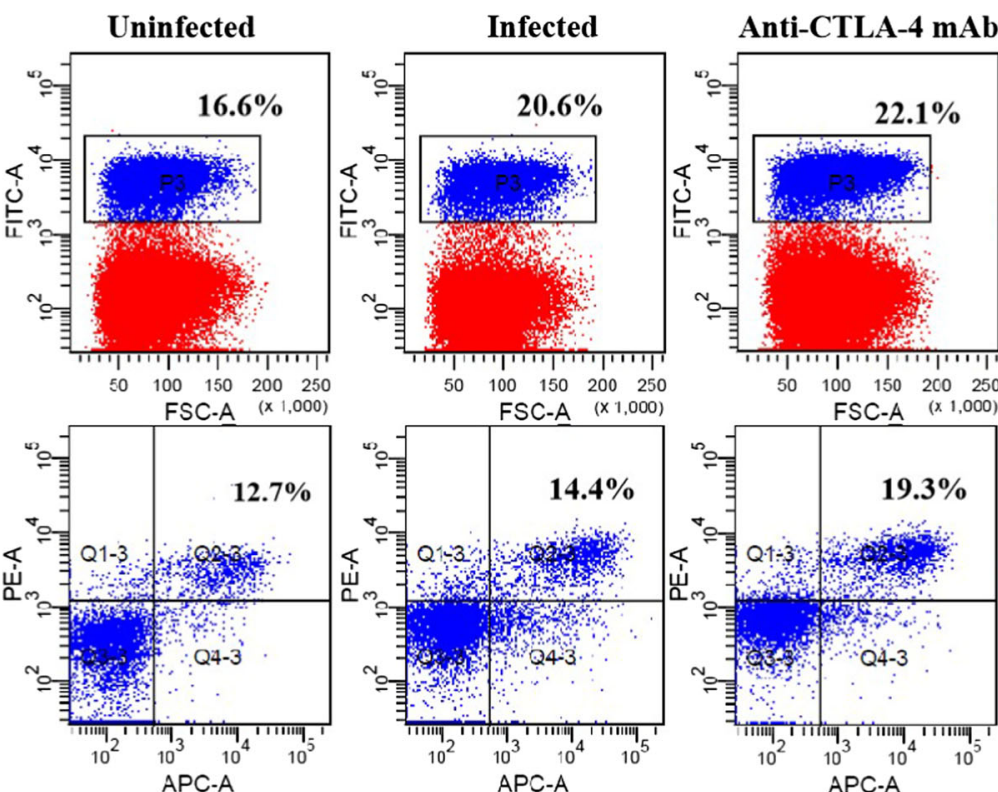

Anti-CTLA-4 mAb
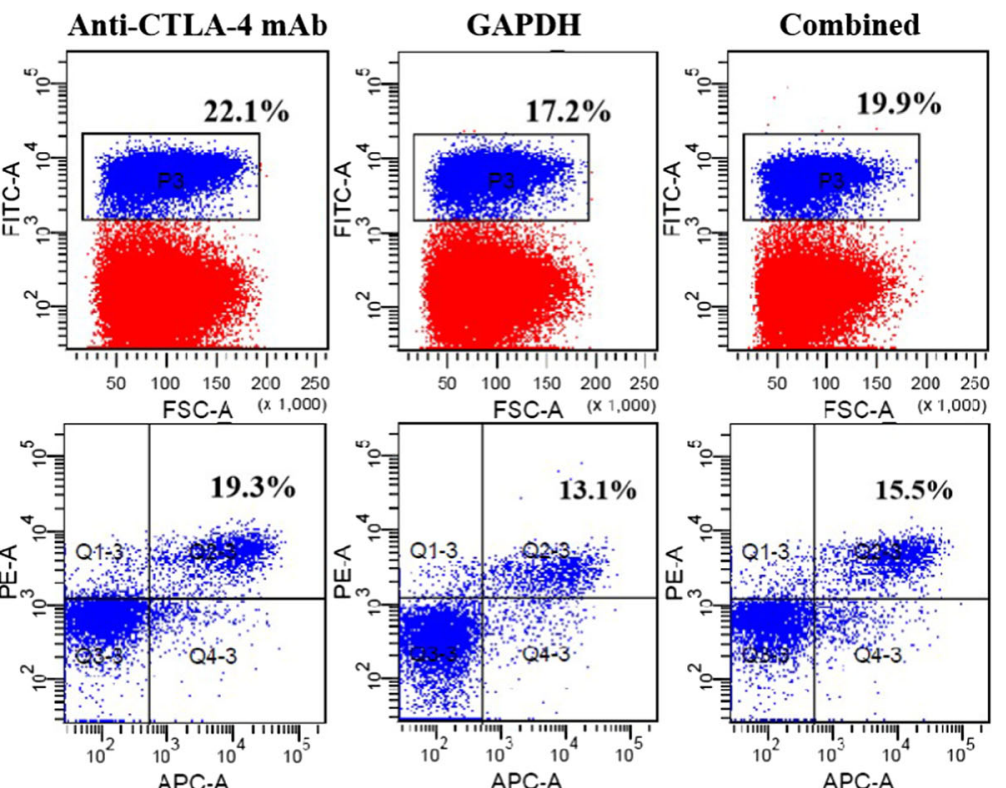

$*$

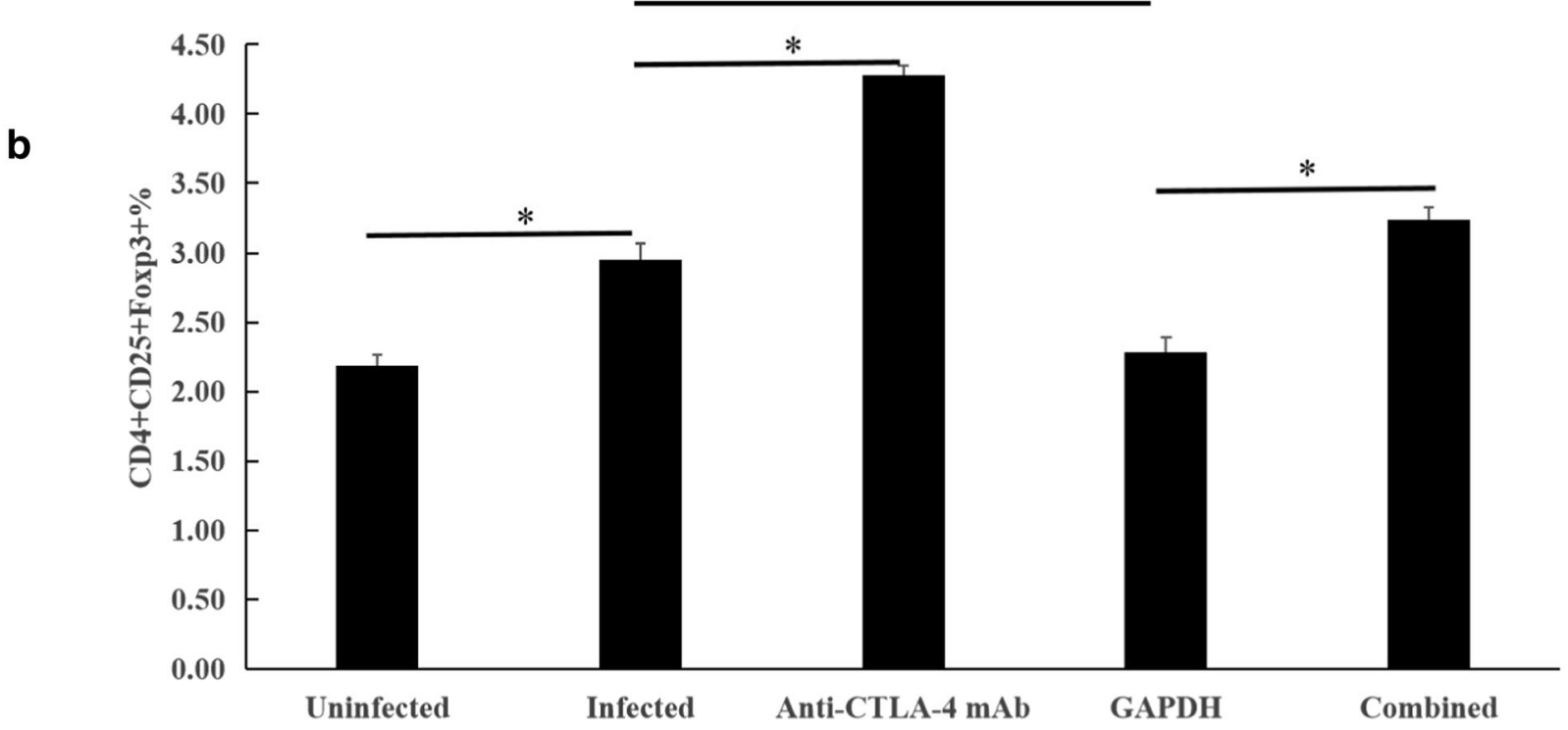

Fig. 2 Percentages of Tregs in splenocytes of mice from a representative flow cytometry result (a) and multigroup comparison (b) for the uninfected, infected control, anti-CTLA-4 mAb, GAPDH, and combined groups. Upper panels, the P3 gate denotes the frequency of
$\mathrm{CD}^{+} \mathrm{T}$ cells among spleen cells. Lower panels, the Q2-3 gate indicates the frequency of CD $25^{+}$Foxp $3^{+}$lymphocytes in the P3 gate. Data are presented as mean $\pm \mathrm{SD}$ for triplicate experiments; $* P<0.05$ 

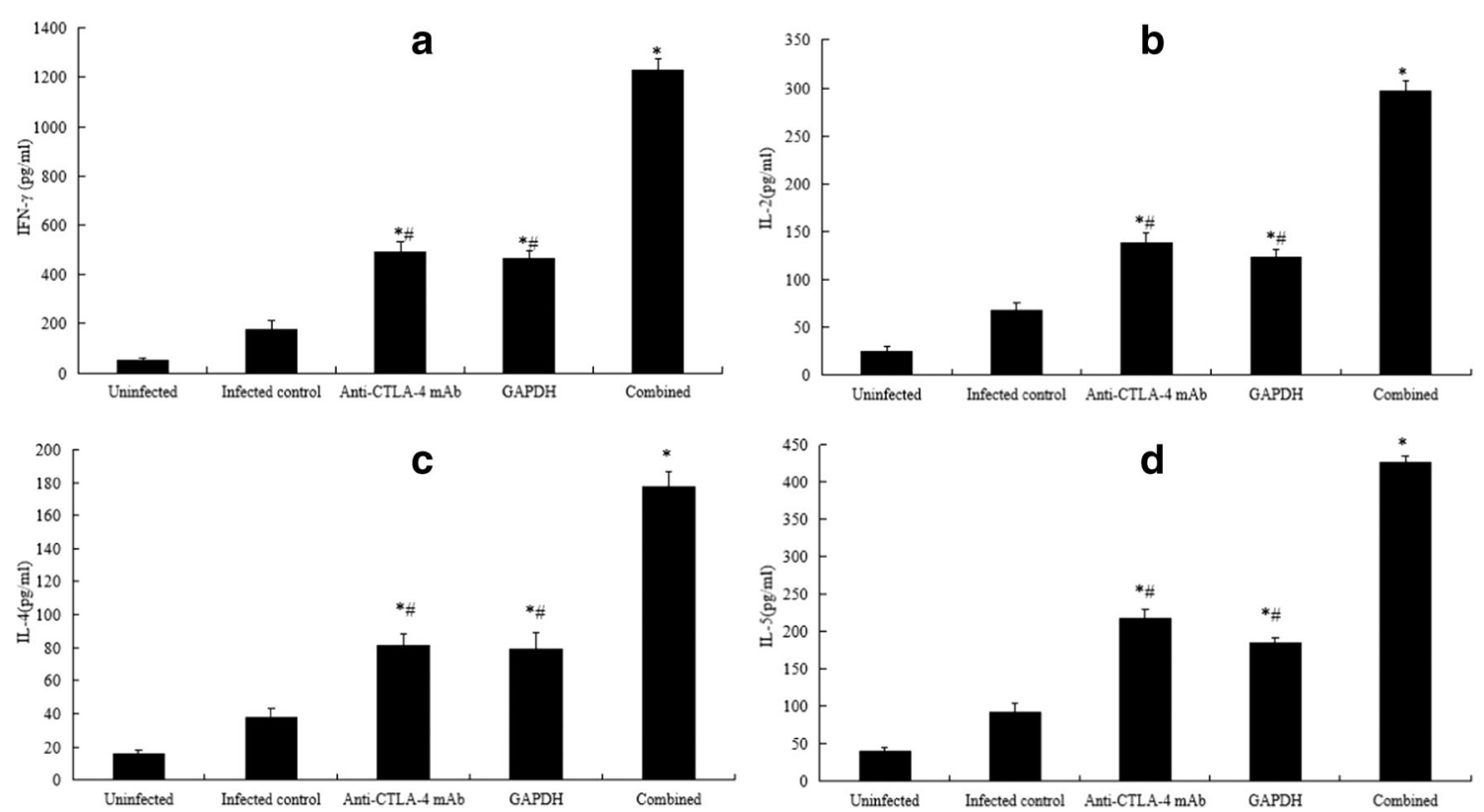

Fig. 3 Contents of IFN- $\gamma(\mathbf{a})$, IL-2 (b), IL-4 (c), and IL-5 (d) in the supernatant of cultured spleen cells measured 5 weeks post-infection. Data represent the mean \pm SD of triplicate experiments. $* P<0.05$ compared to the infected control group; $\# P<0.05$ compared to the combined group

groups showed higher levels of all cytokines, whereas the combined group had highly significantly greater levels.

\section{Histopathological evaluation}

The livers of the four infected groups showed typical egg granuloma. The mean granuloma sizes within the antiCTLA-4 mAb-treated $(286 \pm 41 \mu \mathrm{m})$ and co-treated groups $(317 \pm 56 \mu \mathrm{m})$ were larger than those of the infected control group $(130 \pm 28 \mu \mathrm{m})(P<0.05)$. The size of the granuloma was also larger within the GAPDH group (164 $\pm 31 \mu \mathrm{m}$ ); however, the increase was not statistically significant compared to that of the infected control group. Compared to that of the combination group, the size of the granuloma was significantly smaller in the GAPDH group $(P<0.05)$ (Fig. 4).
Fig. 4 H\&E staining of Schistosoma egg granuloma in the livers of the following four infected groups: Infected group (a), anti-CTLA-4 mAb (b),

GAPDH (c), and combined group (d)
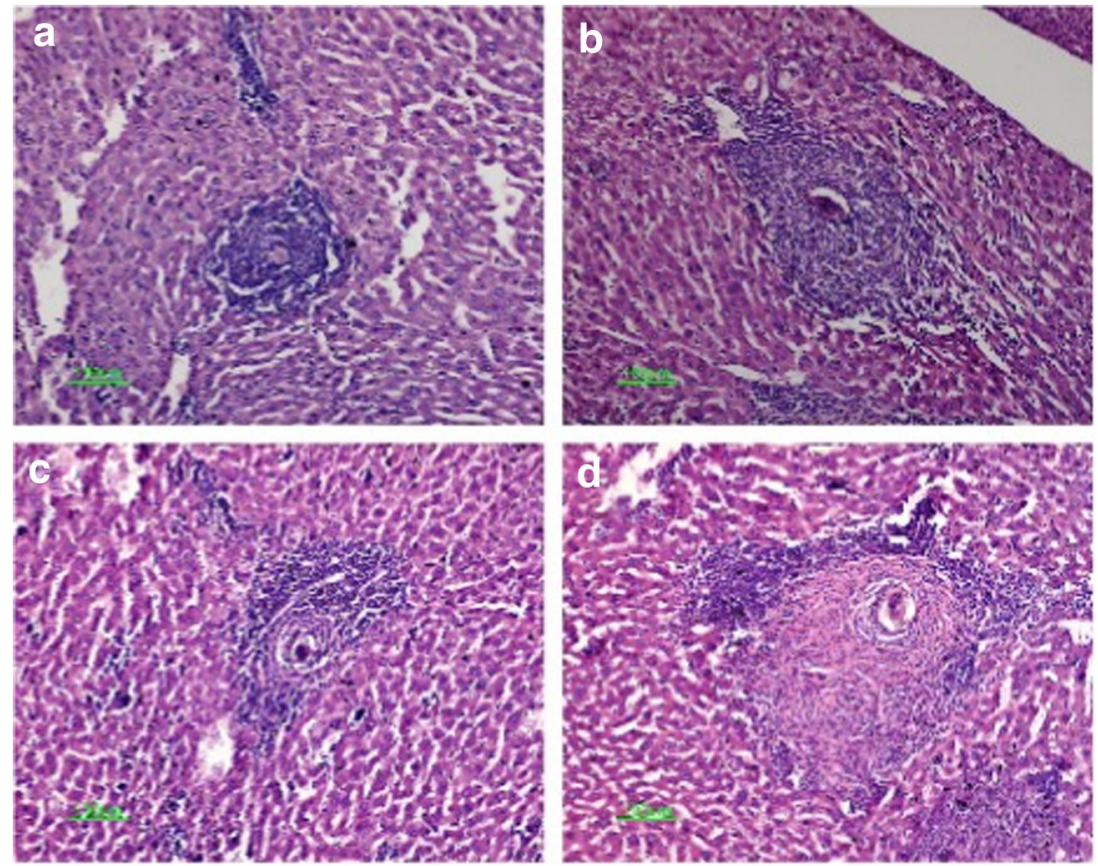


\section{Discussion}

CTLA-4, as a negative regulator of $\mathrm{T}$ cell activation and proliferation, plays an important role in parasitic infection (Mackroth et al. 2016). Walsh et al. (2007) identified marked expansion of the CTLA $-4^{+}$population during S. mansoni infection, which helps it to escape the host immune attack. Therefore, we hypothesized that targeting CTLA- 4 could improve the protective effect of a GAPDH vaccine against $S$. japonicum, which was tested using a mouse model of $S$. japonicum infection. Indeed, we found that the protection conferred by the GAPDH vaccine was promoted by the addition of anti-CTLA- $4 \mathrm{mAb}$ in terms of an almost $30 \%$ further reduction in the worm burden. These results suggest that anti-CTLA-4 mAb may be an effective adjuvant to enhance the immune protective effect of vaccines. Fahmy et al. (2016) reported that the combination of anti-CTLA-4 therapy with a Bacillus Calmette-Guerin vaccine might enhance the immune activity in the bladder submucosal tissue. Although the protective mechanism of GAPDH in the host and the effect of CTLA-4 as an adjuvant are unclear, these effects may involve the regulation of Tregs in which CTLA-4 is constitutively expressed.

Tregs are believed to play a key role in maintaining peripheral immune tolerance, and have been shown to be related to the immune protective effects of the S. japonicum GST vaccine, which induced Tregs, although the protective effect is quite poor (Tang et al. 2017). Here, flow cytometry analysis demonstrated that the frequency of Tregs was reduced in the GAPDH group but was higher in the anti-CTLA-4 group compared to that of the infected control. This suggests that the immunoprotective effect of GAPDH may be related to its downregulation of Tregs, whereas the mechanism by which CTLA-4 antibody enhances these effects of the GAPDH vaccine is Tregs-independent. Consistently, previous studies showed that CTLA-4 blockade increased the number of activated effector $\mathrm{CD} 4+\mathrm{T}$ cells and also increased the number of Tregs (Kavanagh et al. 2008), while elimination of Tregs and CTLA-4 blockade synergistically enhanced specific cytotoxicity (Suarez et al. 2011).

Moreover, the Th1-type response plays an important role in determining the level of protective efficacy (You et al. 2018). Administration of GAPDH and anti-CTLA-4 mAb alone and in combination elevated the levels of Th1 cytokines (IFN- $\gamma$ and IL-2) and Th2 cytokines (IL-4 and IL-5) in infected mice. This suggests that anti-CTLA-4 mAb is beneficial for the host to clear S. japonicum through enhancement of the Th1-type immune response. Collectively, these results help to clarify the complexity of the role of Tregs in schistosomiasis infection and emphasize the special role of CTLA-4 in regulating the Th2 response (Walsh et al. 2007). The pathology caused by $S$. japonicum eggs is also considered to reflect a Th2-type immune response (Wang et al. 2015). Compared with the infected control group, the egg granuloma diameter of the anti-CTLA-4 mAb or combined group increased significantly.
Although anti-CTLA-4 mAb has been reported to have obvious therapeutic effects against worms, it is also associated with damage to the body via inducing immune-related adverse events (Kadono 2017). S. mansoni PIII antigen was capable of modulating the in vitro granuloma reaction, which was found to be related to the balance of CD28, CTLA-4, and CD86 in human schistosomiasis (Zouain et al. 2004). In addition, CTLA-4 was shown to reduce the extent of T cell-mediated parasite tissue sequestration in a mouse model of malaria, thus limiting the immunopathology response (Haque et al. 2010). In line with the present study, anti-CTLA-4 mAb was shown to eliminate $S$. japonicum in a previous schistosomiasis model, but at the expense of increased pathological damage (Tang et al. 2014).

In conclusion, we demonstrate that anti-CTLA-4 mAb could be an effective adjuvant to enhance the protective effect of a GAPDH vaccine against $S$. japonicum via enhancement of Th1-type and Th2-type immune responses but also enhances damage to the host.

Funding This research was funded by Hubei Provincial Planning Commission Joint Fund project (grant number WJ2018H0129), Scientific Research Subject of the Health and Family Planning Commission of Wuhan Municipality (grant number WX17A08), and Hubei Provincial Natural Science Foundation project (grant number 2017CFB570.

Compliance with ethical standards The experiment was approved by the committee on Animal Research of Wuchang Hospital (No. 2017-0028).

Conflict of interest The authors declare that they have no conflict interest.

\section{References}

Álvarez-Larrotta C, Agudelo OM, Duque Y, Gavina K, Yanow SK, Maestre A, Carmona-Fonseca J, Arango E (2019) Submicroscopic Plasmodium infection during pregnancy is associated with reduced antibody levels to tetanus toxoid. Clin Exp Immunol 195(1):96-108

Argiro LL, Kohlstädt SS, Henri SS, Dessein HH, Matabiau VV, Paris PP, Bourgois AA, Dessein AJ (2000) Identification of a candidate vaccine peptide on the $37 \mathrm{kDa}$ Schistosoma mansoni GAPDH. Vaccine 18(19):2039-2048

Cheng YL, Song WJ, Liu WQ, Lei JH, Mo HM, Ruppel A, Li YL (2008) The effects of T cell deficiency on the development of worms and granuloma formation in mice infected with Schistosoma japonicum. Parasitol Res 102(6):1129-1134

Dyck L, Mills KHG (2017) Immune checkpoints and their inhibition in cancer and infectious diseases. Eur J Immunol 47(5):765-779

Fahmy O, Khairul-Asri MG, Stenzl A, Gakis G (2016) Systemic antiCTLA-4 and intravesical Bacille-Calmette-Guerin therapy in nonmuscle invasive bladder cancer: is there a rationale of synergism? Med Hypotheses 92:57-58

Gannavaram S, Bhattacharya P, Ismail N1, Kaul A, Singh R, Nakhasi HL (2016) Modulation of innate immune mechanisms to enhance Leishmania vaccine-induced immunity: role of coinhibitory molecules. Front Immunol 7:187 
Haque A, Best SE, Amante FH, Mustafah S, Desbarrieres L, de Labastida F, Sparwasser T, Hill GR, Engwerda CR (2010) CD4+ natural regulatory $\mathrm{T}$ cells prevent experimental cerebral malaria via CTLA-4 when expanded in vivo. PLoS Pathog 6(12):e1001221

Kadono T (2017) Immune-related adverse events by immune checkpoint inhibitors. Nihon Rinsho Meneki Gakkai Kaishi 40(2):83-89

Kavanagh B, O'Brien S, Lee D, Hou Y, Weinberg V, Rini B, Allison JP, Small EJ, Fong L (2008) CTLA4 blockade expands FoxP3+ regulatory and activated effector CD4+ T cells in a dose-dependent fashion. Blood 112(4):1175-1183

Larsen SK, Munir S, Woetmann A, Frøsig TM, Odum N, Svane IM, Becker JC, Andersen MH (2013) Functional characterization of Foxp3-specific spontaneous immune responses. Leukemia 27(12): 2332-2340

Li MJ, Lei JH, Wang T, Lu SJ, Guan F, Liu WQ, Li YL (2011) Cimetidine enhances the protective effect of GST DNA vaccine against Schistosoma japonicum. Exp Parasitol 128(4):427-432

Mackroth MS, Abel A, Steeg C, Schulze Zur Wiesch J, Jacobs T (2016) Acute malaria induces PD1+CTLA4+ effector T cells with cellextrinsic suppressor function. PLoS Pathog 12(11):e1005909

Mo HM, Liu WQ, Lei JH, Cheng YL, Wang CZ, Li YL (2007) Schistosoma japonicum eggs modulate the activity of CD4+ CD25+ Tregs and prevent development of colitis in mice. Exp Parasitol 116(4):385-389

Perez-Casal J, Potter AA (2016) Glyceradehyde-3-phosphate dehydrogenase as a suitable vaccine candidate for protection against bacterial and parasitic diseases. Vaccine 34(8):1012-1017

Pietruczuk M, Eusebio M, Kraszula L, Kupczyk M, Kuna P (2012) Phenotypic characterization of ex vivo CD4+CD25highCD127low immune regulatory $\mathrm{T}$ cells in allergic asthma: pathogenesis relevance of their FoxP3, GITR, CTLA-4 and FAS expressions. J Biol Regul Homeost Agents 26(4):627-639

Rowshanravan B, Halliday N, Sansom DM (2018) CTLA-4: a moving target in immunotherapy. Blood 131(1):58-67

Ruppel A, Shi YE, Moloney NA (1990) Schistosoma mansoni and S. japonicum: comparison of levels of ultraviolet irradiation for vaccination of mice with cercariae. Parasitology 101(1):23-26

Suarez N, Alfaro C, Dubrot J, Palazon A, Bolaños E, Erro L, HervasStubbs S, Martinez-Forero I, Morales-Kastresana A, Martin-Algarra S, Sangro B, Lecanda F, Perez-Gracia JL, Gonzalez A, Melero I (2011) Synergistic effects of CTLA-4 blockade with tremelimumab and elimination of regulatory $\mathrm{T}$ lymphocytes in vitro and in vivo. Int J Cancer 129(2):374-386
Sun LP, Wang W, Hong QB, Li SZ, Liang YS, Yang HT, Zhou XN (2017) Approaches being used in the national schistosomiasis elimination programme in China: a review. Infect Dis Poverty 6(1):55

Tallima H, Dvořák J, Kareem S, Abou El Dahab M, Abdel Aziz N, Dalton JP, El Ridi R (2017) Protective immune responses against Schistosoma mansoni infection by immunization with functionally active gut-derived cysteine peptidases alone and in combination with glyceraldehyde 3-phosphate dehydrogenase. PLoS Negl Trop Dis 11(3): e0005443

Tang CL, Lei JH, Guan F, Li YL, Liu R, Grevelding CG, Jiang MS (2014) Dong HF (2014) effect of cytotoxic T-lymphocyte-associated protein 4 on CD4(+)CD25(+) regulatory T cells in murine schistosomiasis japonica. Exp Parasitol 136:74-78

Tang CL, Yang J, Cheng LY, Cheng LF, Liu ZM (2017) Anti-CD25 monoclonal antibody enhances the protective efficacy of Schistosoma japonicum GST vaccine via inhibition of CD4+ CD25+Foxp3+ regulatory T cells. Parasitol Res 116(10):2727-2732

Waine GJ, Becker M, Yang W, Kalinna B, McManus DP (1993) Cloning, molecular characterization, and functional activity of Schistosoma japonicum glyceraldehyde-3-phosphate dehydrogenase, a putative vaccine candidate against schistosomiasis japonica. Infect Immun 61(11):4716-4723

Walsh CM, Smith P, Fallon PG (2007) Role for CTLA-4 but not CD25+ $\mathrm{T}$ cells during Schistosoma mansoni infection of mice. Parasite Immunol 29(6):293-308

Wang Y, Zhang J, Yin J, Shen Y, Wang Y, Xu Y, Cao J (2015) The formation of egg granulomas in the spleens of mice with late Schistosoma japonicum infection alters splenic morphology. Parasit Vectors 8:375

Waterhouse P, Penninger JM, Timms E, Wakeham A, Shahinian A, Lee KP, Thompson CB, Griesser H, Mak TW (1995) Lymphoproliferative disorders with early lethality in mice deficient in Ctla-4. Science 270(5238):985-988

You H, Harvie M, Du X, Rivera V, Zhang P, McManus DP (2018) Protective immune responses generated in a murine model following immunization with recombinant Schistosoma japonicum insulin receptor. Int J Mol Sci 19(10):1-16

Zouain CS, Falcão PL, Goes TS, Leite MF, Goes AM (2004) Schistosoma mansoni PIII antigen modulates in vitro granuloma formation by regulating CD28, CTLA-4, and CD86 expression in humans. Immunol Lett 91(2-3):113-118

Publisher's note Springer Nature remains neutral with regard to jurisdictional claims in published maps and institutional affiliations. 